\title{
Orbital Fat Volumetry and Water Fraction Measurements Using T2-Weighted FSE-IDEAL Imaging in Patients with Thyroid-Associated Orbitopathy
}

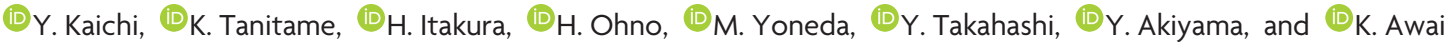

\begin{abstract}
BACKGROUND AND PURPOSE: The quantitative evaluation of orbital fat proliferation and edema and the assessment of extraocular muscles are useful for diagnosing and monitoring thyroid-associated orbitopathy. To evaluate therapy-induced quantitative changes in the orbital fat of patients with thyroid-associated orbitopathy, we performed volumetric and water fraction measurements by using T2-weighted FSE iterative decomposition of water and fat with echo asymmetry and least-squares estimation (FSE-IDEAL) imaging.
\end{abstract}

MATERIALS AND METHODS: Orbital FSE-IDEAL images of 30 volunteers were acquired twice within 1 week. Nine patients with thyroidassociated orbitopathy underwent FSE-IDEAL imaging before and after methylprednisolone pulse therapy, and the treatment results were assessed by using their pre- and post-methylprednisolone pulse therapy clinical activity scores. We performed volumetric and water fraction measurements of orbital fat by using FSE-IDEAL imaging and evaluated interscan differences in the volunteers. In patients with thyroid-associated orbitopathy, we compared pre- and posttherapy orbital fat measurements and assessed the correlation between the pretherapy values and clinical activity score improvement.

RESULTS: The reproducibility of results obtained by the quantitative evaluation of orbital fat in volunteers was acceptable. After methylprednisolone pulse therapy, the water fraction in the orbital fat of patients with thyroid-associated orbitopathy was significantly decreased $(P<.001)$. There was a significant positive correlation between the pretherapy water fraction and clinical activity score improvement (right, $r=0.82$; left, $r=0.79$ ) and a significant negative correlation between the pretherapy volume and clinical activity score improvement (bilateral, $r=-0.84$ ).

CONCLUSIONS: Volumetric and water fraction measurements of orbital fat by using FSE-IDEAL imaging are feasible and useful for monitoring the effects of therapy and for predicting the response of patients with thyroid-associated orbitopathy to methylprednisolone pulse therapy.

ABBREVIATIONS: CAS = clinical activity score; FSE-IDEAL = FSE iterative decomposition of water and fat with echo asymmetry and least-squares estimation; MPPT = methylprednisolone pulse therapy; $\mathrm{SI}=$ signal intensity; TAO = thyroid-associated orbitopathy

T hyroid-associated orbitopathy (TAO) is the most common extrathyroid manifestation of Graves disease. The enlargement of orbital fat and extraocular muscles in the relatively fixed volume space imposed by the bony orbit may produce proptosis, ocular motility loss, and decreased visual acuity. ${ }^{1}$ Histologic studies showed lymphocytic infiltration and edema due to the accu-

Received January 28, 2016; accepted after revision May 8.

From the Department of Diagnostic Radiology (Y.K., K.A.), Graduate School and Institute of Biomedical and Health Sciences, Hiroshima University, Hiroshima, Japan; Department of Radiology (K.T.), Chugoku Rosai Hospital, Kure, Japan; Department of Ophthalmology and Visual Science (H.I.) and Department of Molecular and Internal Medicine (H.O., M.Y.), Institute of Biomedical and Health Sciences, Hiroshima University, Hiroshima, Japan; and Department of Clinical Radiology (Y.T. Y.A.), Hiroshima University Hospital, Hiroshima, Japan.

Please address correspondence to Yoko Kaichi, MD, Diagnostic Radiology, Graduate School of Biomedical and Health Sciences, Hiroshima University, Kasumi 1-2-3, Minami-ku, Hiroshima 734-8551, Japan; e-mail: kaichi@hiroshima-u.ac.jp

http://dx.doi.org/10.3174/ajnr.A4859 mulation of hydrophilic, interstitial glycosaminoglycans in the orbital fat and extraocular muscles, ${ }^{2,3}$ attributable to antigenic cross-reactivity between thyroid proteins and orbital fibroblasts. ${ }^{4}$

The course of TAO can be divided into the active, regressing, and a burnt-out phase. ${ }^{5}$ Early active disease is treated with combined immunosuppression. ${ }^{6,7}$ The identification of patients who stand to benefit from this therapy is important but clinically difficult, despite the availability of activity scoring systems. Consequently, objective methods are needed.

CT has been used to quantify the volume of extraocular muscles and orbital fat in TAO. ${ }^{8-11}$ However, ocular radiation exposure limits its clinical use. Because MR imaging without ionizing radiation yields orbital images with excellent soft-tissue contrast in any plane, it has been used for evaluating TAO. ${ }^{12-15}$

The quantitative evaluation of the orbital fat volume on T1weighted images was reported useful for assessing the severity of $\mathrm{TAO}$ and for monitoring the treatment response. ${ }^{12,16}$ Recently, 

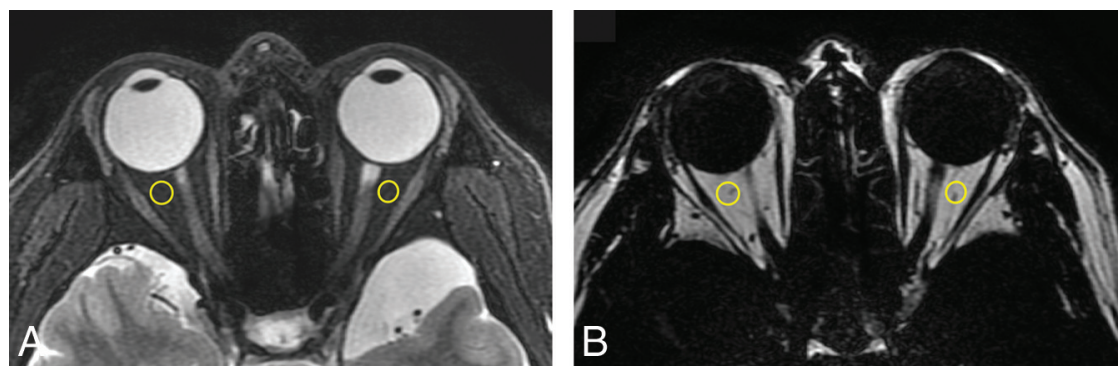

FIG 1. Axial water $(A)$ and fat $(B)$ images of a healthy volunteer. The ROls are encircled in yellow.
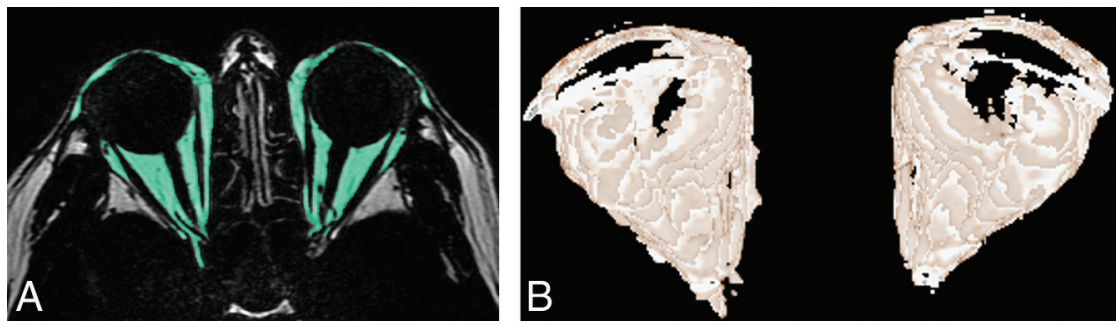

FIG 2. Axial fat image $(A)$ of a healthy volunteer. The selected orbital fat is light green. We separated fat tissue by using an adequate signal-intensity threshold and manually removed extraorbital fat. In this volunteer, the internal rectus muscle showed an average SI of 207 and an SD of 112 , whereas the orbital fat showed an average SI of 1636 and an SD of 88. Therefore the threshold value was $[(207+112)+(1636-88) / 2=933]$. Using FSE-IDEAL images, we then produced $3 \mathrm{D}$ reconstruction images of the bilateral orbital fat $(B)$ and measured the orbital fat volume on a workstation.

Higashiyama et $\mathrm{al}^{17}$ demonstrated that after methylprednisolone pulse therapy (MPPT), the orbital fat volume was unchanged, while the total volume of extraocular muscles was decreased on T2-weighted images. However, in our search of the literature, we found no reports on quantitative changes in orbital fat edema after MPPT or orbital irradiation. Consequently, the correlation between the volume and edema of orbital fat and the treatment response of patients with TAO remains to be elucidated.

FSE iterative decomposition of water and fat with echo asymmetry and least-squares estimation (FSE-IDEAL), a novel 3-point Dixon method, is useful for separating the fat signal from the water signal ${ }^{18,19}$ but has not been used to quantify the orbital structures in TAO. To evaluate the feasibility of FSE-IDEAL imaging for the precise quantitative evaluation of orbital fat, we subjected healthy volunteers to 2 FSE-IDEAL imaging studies performed during 1 week. We measured the volume and the water fraction of their orbital fat and ascertained the reproducibility of the measurement results. We also acquired FSE-IDEAL images in patients with TAO to assess MPPT-induced changes in the volume and water fraction of their orbital fat and evaluated the correlation between the quantitative values of orbital fat and the improvement in their symptoms.

\section{MATERIALS AND METHODS \\ Subjects}

This prospective study was approved by the Ethics Committee of Hiroshima University. Informed consent was obtained from all participants before entry into the study.

We recruited 30 healthy volunteers ( 15 men, 15 women; median age, $29 \pm 7.6$ years). They underwent orbital FSE-IDEAL imaging twice within 1 week to evaluate the reproducibility of the water fraction and the volume measurements of the orbital fat.
We also recruited 16 patients older than 20 years of age who had a clinical diagnosis of TAO between December 2013 and November 2015. The recorded activity of their TAO was based on their clinical activity score (CAS), including pain, eyelid erythema or edema, conjunctival hyperemia and chemosis, and a swollen caruncle. ${ }^{20}$ All 16 patients underwent orbital FSE-IDEAL imaging. Two of them underwent local injection of triamcinolone acetonide into the orbit, and 5 of them followed a conservative wait-and-see approach because their TAO was slight and the risk of MPPT would exceed the benefit. Consequently, the 9 patients ( 2 men, 7 women; median age, $57 \pm 12.5$ years; 18 eyes $)$ who received MPPT (500 mg of methylprednisolone administered intravenously once a day for 3 consecutive days) were included in this study and underwent FSE-IDEAL imaging again $1.6 \pm 0.7$ months thereafter. Re-evaluation of the TAO activity was based on the latest CAS obtained around the time of the second MR imaging study (the interval, 1-15 days; median, 6.5 days).

\section{MR Imaging}

All images were acquired on a 3T scanner (Signa Excite HD 3.0; GE Healthcare, Milwaukee, Wisconsin; gradient strength, 40 $\mathrm{mT} / \mathrm{m}$; slew rate, $150 \mathrm{~T} / \mathrm{m} / \mathrm{s}$ ) by using an 8 -channel phased array brain coil. We optimized the T2-weighted FSE-IDEAL sequence (TR/TE, $6000 / 100 \mathrm{~ms}$; flip angle, $90^{\circ}$; image matrix, $288 \times 160$; FOV, $160 \times 160 \mathrm{~mm}$; section thickness/gap, $2 / 0 \mathrm{~mm}$; asymmetric echo shifts, $-\pi / 6, \pi / 2,7 \pi / 6$; number of acquisitions, 3 ; number of sections, 32; scan time, 2 minutes 42 seconds) and obtained orbital water and fat images for all subjects.

\section{Orbital Fat Water Fraction and Volume Measurements}

We placed an ROI in the orbital fat on FSE-IDEAL images of water and fat and measured the average signal intensity (SI) in the ROIs. Next, we defined and calculated the water fraction of the orbital fat as [SI Water / (SI Water + SI Fat)] to assess the orbital fat edema on the basis of the fat fraction (SI Fat / [SI Water + SI Fat]), ${ }^{18}$ defined to quantify fatty infiltration (Fig 1 ). The orbital fat volume of both eyes was measured on a workstation (Virtual Place Raijin; AZE Ltd, Tokyo, Japan). We first separated fat tissue from other structures by using the threshold value, recorded as the mean value between the average SI plus the SD of the ROI in the internal rectus muscle and the average SI minus the SD of the ROI in the orbital fat, considering some dispersion of signal intensities in the ROIs. Then we manually removed the fatty marrow of the orbital bone and outer fat; the orbital fat volume was automatically measured on the workstation (Fig 2). 


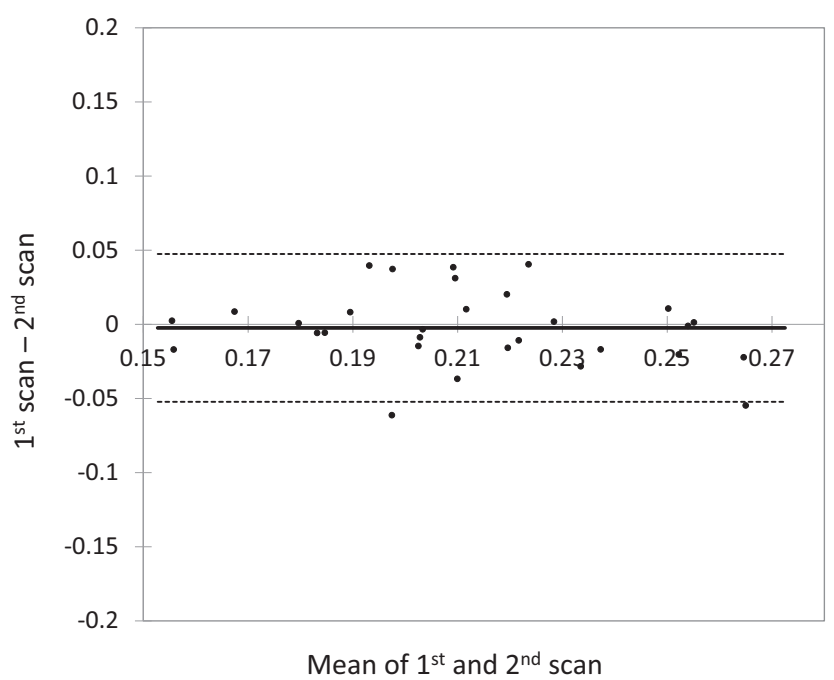

A

- Bias $\quad$-..-.... Cl $(95 \%)$

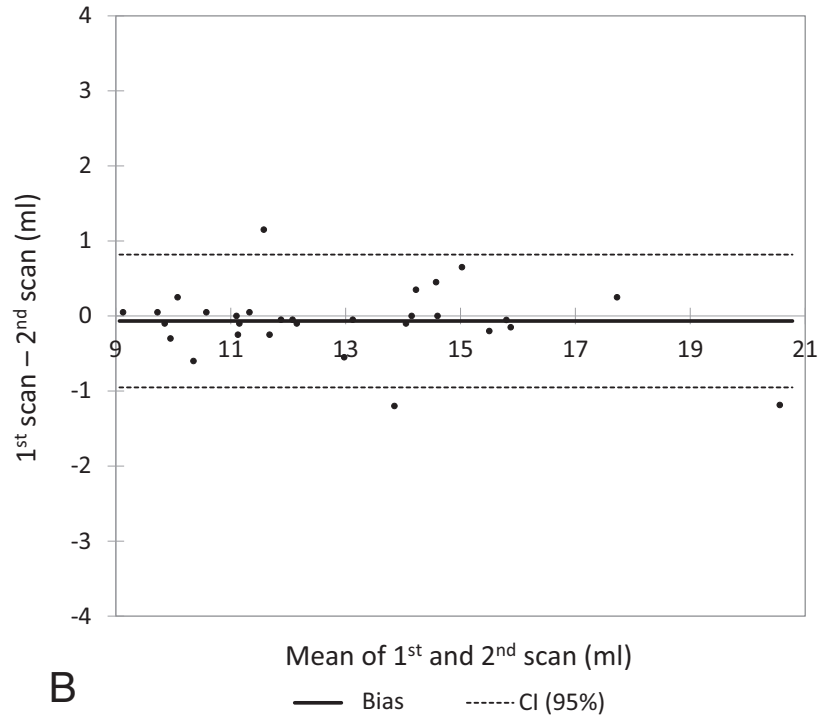

FIG 3. Bland-Altman analysis confirming the interscan reproducibility of the water fraction $(A)$ and the volume of orbital fat (B) on FSE-IDEAL images of the volunteers.

\section{Statistical Analyses}

All statistical analyses were performed with commercially available software (XLSTAT, version 2015.6.01.244, Addinsoft; https://www.xlstat.com/en/company/about-us) and Excel 2010 (Microsoft, Redmond, Washington).

We used Bland-Altman analysis and the Pearson correlation coefficient to evaluate interscan differences in the volunteers and used the mean value of the water fraction and the volume of the bilateral orbit in our analyses.

In patients with $\mathrm{TAO}$, we performed paired-sample $t$ tests to compare the water fraction and the volume of orbital fat before and after MPPT. The right and left orbits were examined separately because patients with unilateral TAO manifested unilateral excess fat exophthalmos. ${ }^{10}$ To ascertain that the values obtained before and after treatment were significantly different, we applied the 2-sample $t$ test to compare the therapy-induced changes with the values obtained in the volunteers. We defined the therapeutic effect of MPPT as the following formula: [CAS Improvement Ratio $=$ (Pretherapy CAS - Posttherapy CAS) / Pretherapy CAS)] and determined the correlation between the measured values before MPPT and the CAS improvement ratio with the Pearson correlation coefficient test. Differences of $P<.05$ were considered statistically significant.

\section{RESULTS}

\section{Volunteers}

The difference in the water fraction and the volume of orbital fat on the first and second scans was $1.1 \% \pm 11.9 \%$ and $0.5 \% \pm$ $3.6 \%$, respectively. Bland-Altman analysis of the measurement results showed that their reproducibility was adequate (water fraction: $r=0.71$, bias $=-0.002 ; 95 \%$ CI bias, $-0.012-0.007$; 95\% CI, $-0.052-0.047$; volume: $r=0.99$, bias $=-0.066$; $95 \% \mathrm{CI}$ bias, $-0.235-0.102$; 95\% CI, $-0.951-0.819$ ) (Fig 3).

\section{Patients}

The clinical characteristics of the 9 patients with TAO are summarized in the Table. After MPPT, the water fraction in the orbital fat on both sides was significantly decreased (right,
Patient profiles

\begin{tabular}{lc}
\multicolumn{1}{c}{ Characteristics } \\
\hline Median age (yr) (range) & $57(43-82)$ \\
Sex: male/female & $2 / 7$ \\
Median duration of the treatment for GD (mo) (range) & $11(1-168)$ \\
Median duration from the onset of TAO (mo) (range) & $4(1-8)$ \\
Smoking: yes/no & $3 / 6$ \\
Median pretherapy CAS (range) & $2(1-4)$ \\
Involvement of extraocular muscle: yes/no & $7 / 2$ \\
\hline
\end{tabular}

Note:-GD indicates Graves disease.

$12.4 \% \pm 3.8 \%, P<.001$; left, $13.6 \% \pm 6.5 \%, P<.001$; Fig $4 A)$. The treatment-induced reduction in the water fraction was significantly larger than the interscan difference observed in the volunteers (right, $P=.002$; left, $P=.001$ ). There was no significant difference in the orbital fat volume pre- and posttreatment (right, $2.0 \% \pm 7.8 \%, P=.37$; left, $1.9 \% \pm 3.8 \%$, $P=.17$, Fig 4B).

The pre- and posttreatment CAS fell by 3 points in 2 patients, by 2 points in 1 patient, and by 1 point in 3 patients. In the other 3 , there was no change. The positive correlation between the pretreatment water fraction and the CAS improvement rate (right, $r=0.82, P=.007$; left, $r=0.79, P=.012$ ) showed that the higher the pretreatment water fraction, the greater was the posttreatment CAS improvement. On the other hand, there was a negative correlation between the fat volume and CAS improvement (right, $r=$ $-0.84, P=.005$; left, $r=-0.84, P=.005)$, indicating that the larger the pretreatment orbital fat volume, the lower the posttreatment CAS improvement rate.

\section{DISCUSSION}

To the best of our knowledge, this is the first quantitative evaluation of orbital fat by using FSE-IDEAL imaging. We document an MPPT-induced reduction in the water fraction of orbital fat in patients with $\mathrm{TAO}$ and report the positive correlation between the pretreatment water fraction and the 
right, $\mathrm{p}<0.001$

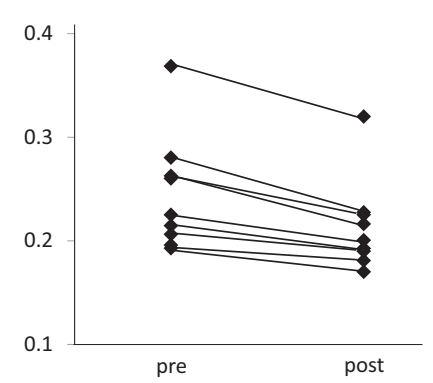

A left, $p<0.001$

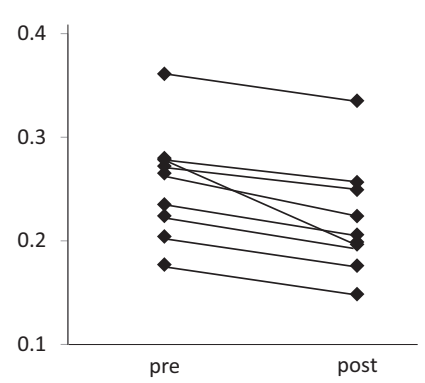

right, $p=0.37$

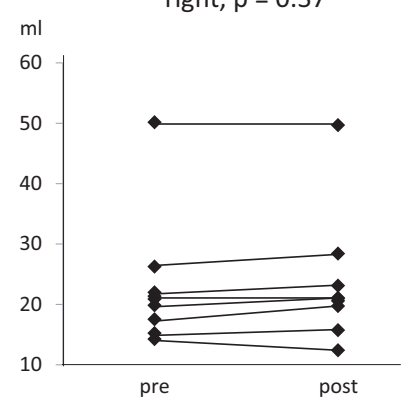

Left, $p=0.17$

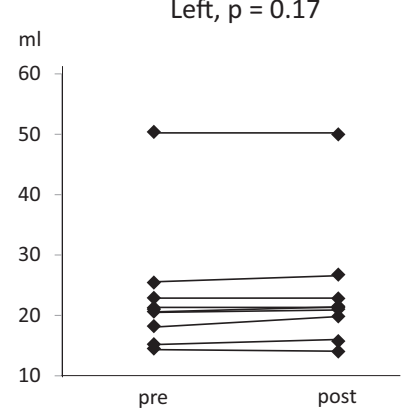

FIG 4. Comparison of the pre- and posttreatment water fraction $(A)$ and the volume of orbital fat $(B)$ in patients with TAO. Methylprednisolone pulse therapy significantly decreased the water fraction. The volume was unchanged.

CAS improvement rate and the negative correlation between the pretreatment orbital fat volume and the CAS improvement rate.

We used FSE-IDEAL imaging to acquire axial images of the bilateral orbits and measured the water fraction and the volume of orbital fat in healthy volunteers and patients with TAO. Although many images contained motion artifacts due to voluntary and involuntary eye movements, it was possible to differentiate the SI of orbital fat and other intraorbital structures by applying an adequate threshold in the orbital fat volumetry. To measure the water fraction in orbital fat, we placed ROIs on the intraorbital fat; measurement of the SI was not hampered by motion artifacts. The reproducibility of water fraction and fat volume measurements on all iteratively acquired FSE-IDEAL images was sufficient.

Others ${ }^{12,21}$ used a $1.5 \mathrm{~T}$ MR imaging scanner and a receiver surface coil to measure orbital fat volumes. Although surface coils can yield orbital images with high spatial resolution, the signal strength decreases as the distance from the coil increases. Our use of a 3T MR imaging scanner and an 8-channel brain coil resulted in orbital images with a high enough signal-to-noise ratio for the estimation of the orbital fat volume and the water fraction.

Orbital images with high spatial and temporal resolution and fewer motion artifacts due to eye movement can be acquired on multidetector row CT scanners. ${ }^{11}$ However, the low soft-tissue contrast on multidetector row CT images makes it difficult to separate out orbital fat. In addition, ocular radiation exposure is a serious concern. Therefore, MR imaging with high soft-tissue contrast is safer for orbital imaging.

We found that MPPT reduced the water fraction in the orbital fat of patients with TAO, possibly because it decreased the severity of edema. Earlier studies ${ }^{7,22-25}$ reported that the SI of extraocular muscles on short $\tau$ inversion recovery sequences correlated well with the disease activity assessed with clinical methods. The correlation was maintained as disease activity changed. Although the reversibility of the relaxation time in muscles with prolonged $\mathrm{T} 2$ values was explained as a therapy-induced decrease in the water content, ${ }^{8}$ treatment-induced changes in orbital fat have not been documented.

Like Higashiyama et al, ${ }^{17}$ we detected no significant difference in the pre- and posttreatment orbital fat volume. Others ${ }^{26-28}$ found no difference in the degree of exophthalmos, which is related to an increase in orbital fat, in patients with TAO who had undergone MPPT. Expansion of the adipose tissue volume is elicited by glycosaminoglycan-related edema and the emergence of a population of newly differentiated fat cells in these tissues. ${ }^{29}$ Although MPPT can reduce orbital fat edema, the increase in the number of fat cells may persist after therapy.

In Japan, MPPT is generally applied in patients with TAO with low CAS scores because the Japanese patients with TAO often have orbital inflammation despite low CAS scores. ${ }^{30}$ We found that the higher the pretreatment water fraction, the greater the posttreatment decrease in the CAS, despite the low pretreatment CAS scores of our study patients. An increase in the water fraction is indicative of an increase in the tissue water content and may reflect acute inflammatory changes. Earlier studies on extraocular muscles found a correlation between the SI increase on pretreatment STIR images and a good therapeutic response $e^{31-33}$ and between prolonged pretreatment $\mathrm{T} 2$ values and a good response to systemic corticosteroids or orbital radiation therapy. ${ }^{33}$ These findings indicate that treatment at an early and immunologically active stage of orbitopathy is important. ${ }^{11,34,35}$ Our quantitative MR imaging study by using the FSE-IDEAL sequence showed that MPPT was useful in patients with TAO whose orbital fat contained an elevated water fraction. On the other hand, the larger the pretreatment orbital fat volume, the lower the posttreatment CAS improvement rate. Because a longer duration of TAO is associated with a larger orbital fat mass and fibrosis, ${ }^{36}$ we think that a response to steroids is less likely when the disease is in its late, inactive stage with more fibrosis. $^{33,34}$

Our study has several limitations. The number of patients with TAO who underwent MPPT was small, and the lack of agematched controls limits the comparison between patients with TAO and healthy individuals. In addition, we used the T2weighted FSE-IDEAL sequence provided by GE Healthcare. It is a novel, 3-point Dixon method that applies iterative algorithms and the region-growing technique to estimate local field inhomogeneities. ${ }^{37-39}$ Application of the 3-point Dixon method developed by different vendors may result in significantly different measurement results with respect to the water fraction of orbital fat. In addition, the demarcation between orbital and upper or lower 
eyelid fat is ambiguous, and this feature may introduce some interobserver variability in the orbital fat volume. Last, the correct cutoff value for the water fraction and the volume of the orbital fat between patients with TAO and their controls remains to be determined.

\section{CONCLUSIONS}

The estimation of the water fraction and the volume of orbital fat on FSE-IDEAL images is feasible, and the reproducibility of these measurements is adequate. FSE-IDEAL imaging is useful for monitoring the effects of therapy and for predicting the response of patients with TAO to methylprednisolone pulse therapy.

Disclosures: Kazuo Awai-UNRELATED: Consultancy: GE Healthcare; Grants/ Grants Pending: Toshiba Medical Systems, ${ }^{*}$ Hitachi Medical Corporation, ${ }^{*}$ Bayer Yakuhin, ${ }^{*}$ Eizai, ${ }^{*}$ Daiichi Sankyo, ${ }^{*}$ Comments: research grants. ${ }^{*}$ Money paid to the institution.

\section{REFERENCES}

1. Kendler DL, Lippa J, Rootman J. The initial clinical characteristics of Graves' orbitopathy vary with age and sex. Arch Ophthalmol 1993; 111:197-201 CrossRef Medline

2. Winand RJ, Cornet G, Etienne-Decerf $J$, et al. Original acquisition in the pathogenesis and the treatment of endocrine ophthalmopathy. Metab Pediatr Syst Ophthalmol 1988;11:126-32 Medline

3. Kahaly G, Hansen C, Beyer J, et al. Plasma glycosaminoglycans in endocrine ophthalmopathy. J Endocrinol Invest 1994;17:45-50 CrossRef Medline

4. Ludgate M, Baker G. Unlocking the immunological mechanisms of orbital inflammation in thyroid eye disease. Clin Exp Immunol 2002; 127:193-98 CrossRef Medline

5. Rundle FF, Wilson CW. Development and course of exophthalmos and ophthalmoplegia in Graves' disease with special reference to the effect of thyroidectomy. Clin Sci 1945;5:177-94 Medline

6. Claridge KG, Ghabrial R, Davis G, et al. Combined radiotherapy and medical immunosuppression in the management of thyroid eye disease. Eye 1997;11:717-22 CrossRef Medline

7. Mayer EJ, Herdman G, Burnett C, et al. Serial STIR magnetic resonance imaging correlates with clinical score of activity in thyroid disease. Eye 2001;15:313-18 CrossRef Medline

8. Kahaly GJ. Imaging in thyroid-associated orbitopathy. Eur J Endocrinol 2001;145:107-18 CrossRef Medline

9. Feldon SE, Lee CP, Muramatsu SK, et al. Quantitative computed tomography of Graves' ophthalmopathy: extraocular muscle and orbital fat in development of optic neuropathy. Arch Ophthalmol 1985;103:213-15 CrossRef Medline

10. Peyster RG, Ginsberg F, Silber JH, et al. Exophthalmos caused by excessive fat: $C T$ volumetric analysis and differential diagnosis. $A J R$ Am J Roentgenol 1986;146:459-64 CrossRef Medline

11. Fang ZJ, Zhang JY, He WM. CT features of exophthalmos in Chinese subjects with thyroid-associated ophthalmopathy. Int JOphthalmol 2013;6:146-49 CrossRef Medline

12. Nishida Y, Tian S, Isberg B, et al. Significance of orbital fatty tissue for exophthalmos in thyroid-associated ophthalmopathy. Graefes Arch Clin Exp Ophthalmol 2002;240:515-20 CrossRef Medline

13. Kvetny J, Puhakka KB, Rohl L. Magnetic resonance imaging determination of extraocular eye muscle volume in patients with thyroid-associated ophthalmopathy and proptosis. Acta Ophthalmol Scand 2006;84:419-23 CrossRef Medline

14. Prummel MF, Gerding MN, Zonneveld FW, et al. The usefulness of quantitative orbital magnetic resonance imaging in Graves' ophthalmopathy. Clin Endocrinol (Oxf) 2001;54:205-09 CrossRef Medline

15. Mayer EJ, Fox DL, Herdman G, et al. Signal intensity, clinical activity and cross-sectional areas on MRI scans in thyroid eye disease. Eur J Radiol 2005;56:20-24 CrossRef Medline

16. Comerci M, Elefante A, Strianese D, et al. Semiautomatic regional segmentation to measure orbital fat volumes in thyroid-associated ophthalmopathy: a validation study. Neuroradiol J 2013;26:373-79 CrossRef Medline

17. Higashiyama T, Nishida Y, Ohji M. Changes of orbital tissue volumes and proptosis in patients with thyroid extraocular muscle swelling after methylprednisolone pulse therapy. Jpn J Ophthalmol 2015;59:430-35 CrossRef Medline

18. Hu HH, Kim HW, Nayak KS, et al. Comparison of fat-water MRI and single-voxel MRS in the assessment of hepatic and pancreatic fat fractions in humans. Obesity (Silver Spring) 2010;18:841-47 CrossRef Medline

19. Takasu M, Tani C, Sakoda Y, et al. Iterative decomposition of water and fat with echo asymmetry and least-squares estimation (IDEAL) imaging of multiple myeloma: initial clinical efficiency results. Eur Radiol 2012;22:1114-21 CrossRef Medline

20. Bartalena L, Baldeschi L, Dickinson A, et al; European Group on Graves' Orbitopathy (EUGOGO). Consensus statement of the European Group on Graves' Orbitopathy (EUGOGO) on management of GO. Eur J Endocrinol 2008;158:273-85 CrossRef Medline

21. Tian S, Nishida Y, Isberg B, et al. MRI measurements of normal extraocular muscles and other orbital structures. Graefes Arch Clin Exp Ophthalmol 2000;238:393-404 CrossRef Medline

22. Hoh HB, Laitt RD, Wakely C, et al. The STIR sequence MRI in the assessment of extraocular muscles in thyroid eye disease. Eye 1994; 8:506-10 CrossRef Medline

23. Laitt RD, Hoh B, Wakeley C, et al. The value of the short tau inversion recovery sequence in magnetic resonance imaging of thyroid eye disease. Br J Radiol 1994;67:244-47 CrossRef Medline

24. Bailey CC, Kabala J, Laitt R, et al. Magnetic resonance imaging in thyroid eye disease. Eye 1996;10:617-19 CrossRef Medline

25. Higashiyama T, Nishida $Y$, Morino K, et al. Use of MRI signal intensity of extraocular muscles to evaluate methylprednisolone pulse therapy in thyroid-associated ophthalmopathy. Jpn J Ophthalmol 2015;59:124-30 CrossRef Medline

26. Bartalena L, Baldeschi L, Dickinson AJ, et al. Consensus statement of the European Group on Graves' Orbitopathy (EUGOGO) on management of Graves' orbitopathy. Thyroid 2008;18:333-46 CrossRef Medline

27. Kauppinen-Mäkelin R, Karma A, Leinonen E, et al. High dose intravenous methylprednisolone pulse therapy versus oral prednisone for thyroid-associated ophthalmopathy. Acta Ophthalmol Scand 2002;80:316-21 CrossRef Medline

28. van Geest RJ, Sasim IV, Koppeschaar HP, et al. Methylprednisolone pulse therapy for patients with moderately severe Graves' orbitopathy: a prospective, randomized, placebo-controlled study. Eur J Endocrinol 2008;158:229-37 CrossRef Medline

29. Bahn RS, Heufelder AE. Pathogenesis of Graves' ophthalmopathy. N Engl J Med 1993;329:1468-75 CrossRef Medline

30. Watanabe N, Noh JY, Kozaki A, et al. Radioiodine-associated exacerbation of Graves' orbitopathy in the Japanese population: randomized prospective study. J Clin Endocrinol Metab 2015;100: 2700-08 CrossRef Medline

31. Hiromatsu $\mathrm{Y}$, Kojima K, Ishisaka N, et al. Role of magnetic resonance imaging in thyroid-associated ophthalmopathy: its predictive value for therapeutic outcome of immunosuppressive therapy. Thyroid 1992;2:299-305 CrossRef Medline

32. Just M, Kahaly GJ, Higer HP, et al. Graves ophthalmopathy: role of MR imaging in radiation therapy. Radiology 1991;179:187-90 CrossRef Medline

33. Ohnishi T, Noguchi S, Murakami N, et al. Extraocular muscles in 
Graves ophthalmopathy: usefulness of T2 relaxation time measurements. Radiology 1994;190:857-62 CrossRef Medline

34. Kendall-Taylor P, Crombie AL, Stephenson AM, et al. Intravenous methylprednisolone in the treatment of Graves' ophthalmopathy. BMJ 1988;297:1574-78 CrossRef Medline

35. Bartalena L, Marcocci C, Bogazzi F, et al. Use of corticosteroids to prevent progression of Graves' ophthalmopathy after radioiodine therapy for hyperthyroidism. N Engl J Med 1989;321:1349-52 CrossRef Medline

36. Potgieser PW, Wiersinga WM, Regensburg NI, et al. Some studies on the natural history of Graves' orbitopathy: increase in orbital fat is a rather late phenomenon. Eur J Endocrinol 2015;173:149-53 CrossRef Medline

37. Costa DN, Pedrosa I, McKenzie C, et al. Body MRI using IDEAL. AJR Am J Roentgenol 2008;190:1076-84 CrossRef Medline

38. Gerdes CM, Kijowski R, Reeder SB. IDEAL imaging of the musculoskeletal system: robust water fat separation for uniform fat suppression, marrow evaluation, and cartilage imaging. AJR Am J Roentgenol 2007;189:284-91 Medline

39. Humbert IA, Reeder SB, Porcaro EJ, et al. Simultaneous estimation of tongue volume and fat fraction using IDEAL-FSE. J Magn Reson Imaging 2008;28:504-08 CrossRef Medline 